\title{
Gross Motor Development of Children with Congenital Heart Disease Receiving Early Systematic Surveillance and Individualized Intervention: Brief Report
}

Solène Fourdain, ${ }^{\mathrm{a}, \mathrm{b}}$ Marie-Noëlle Simard,${ }^{\mathrm{a}, \mathrm{c}, \mathrm{d}}$ Lynn Dagenais, ${ }^{\mathrm{d}}$ Manuela Materassi, ${ }^{\mathrm{d}}$ Amélie Doussau, ${ }^{\mathrm{d}}$ Justine Goulet, ${ }^{\mathrm{b}}$ Karine Gagnon, ${ }^{\mathrm{d}}$ Joëlle Prud'homme, ${ }^{\mathrm{d}}$ Marie-Claude Vinay, ${ }^{\mathrm{d}}$ Mathieu Dehaes, ${ }^{\mathrm{a}, \mathrm{d}, \mathrm{e}, \mathrm{f}}$ Ala Birca, ${ }^{\mathrm{a}, \mathrm{d}, \mathrm{f}}$ Nancy C. Poirier, ${ }^{\mathrm{a}, \mathrm{d}, \mathrm{f}}$ Lionel Carmant, ${ }^{\mathrm{a}, \mathrm{d}, \mathrm{f}}$ Anne Gallagher ${ }^{\mathrm{a}, \mathrm{b}, \mathrm{d} *}$ on behalf of the CINC interdisciplinary team ${ }^{\mathrm{d}}$

${ }^{\text {a }}$ CHU Sainte-Justine Research Centre, Montreal, Quebec, Canada;

${ }^{\mathrm{b}}$ Department of Psychology, University of Montreal, Montreal, Quebec, Canada

${ }^{c}$ School of Rehabilitation, University of Montreal, Montreal, Quebec, Canada;

${ }^{\mathrm{d}}$ Clinique d'Investigation Neurocardiaque (CINC), CHU Sainte-Justine, Montreal, Quebec, Canada;

${ }^{\mathrm{e}}$ Department of Radiology, Radio-oncology and Nuclear Medicine, University of Montreal, Montreal, Quebec, Canada;

${ }_{\mathrm{f}}^{\mathrm{f}}$ Faculty of Medicine, University of Montreal, Montreal, Quebec, Canada

*Corresponding author: Anne Gallagher, Ph.D., anne.gallagher@umontreal.ca CHU Sainte-Justine Research Centre, 3175 Chemin de la Côte Sainte-Catherine, Montreal, Quebec, H3T 1C5 Canada

\begin{abstract}
$\underline{\text { Abstract }}$
Objective: In this pilot study, we described the gross motor development of infants aged 4 to 24 months with congenital heart disease (CHD) and assessed through a systematic developmental screening programme, with individualised motor interventions. Methods: Thirty infants who had cardiac repair underwent gross motor evaluations using the AIMS at 4 months, and the Bayley-III at 12 and 24 months. Results: Based on AIMS, 80\% of 4-month-old infants had a delay in gross motor development and required physical therapy. Gross motor abilities significantly improved by 24 months. Infants who benefited from regular physiotherapy tended to show better improvement in motor scores. Conclusion: Our study highlights the importance of early motor screening in infants with CHD and suggests a potential benefit of early physical therapy in those at-risk. Further research is needed to assess the effectiveness of systematic developmental screening and individualized intervention programmes at identifying at risk patients, and their impact on developmental outcomes.
\end{abstract}

Keywords: congenital heart disease; gross motor development; early intervention; Alberta Infant Motor Scales (AIMS); Bayley Scales of Infant and Toddler Development; Third edition (BayleyIII)
Abbreviations
CHD, Congenital heart disease
AIMS, Alberta Infant Motor Scales
Bayley-III, Bayley Scales of Infant and Toddler Development, Third edition
Bayley-III/GM, gross motor scale of the Bayley Scales of Infant and Toddler Development,
Third edition 


\section{Introduction}

Children with congenital heart disease (CHD) requiring early cardiac surgery experience a broad spectrum of neurodevelopmental disorders characterized by a combination of mild to moderate motor, cognitive, and language impairments. ${ }^{1-10}$ During infancy, gross motor delays typically appear as the primary manifestations of altered neurodevelopment. ${ }^{2,4,5,9,11}$ Longitudinal studies investigating motor development in children with CHD revealed a delay in the acquisition of gross motor skills as early as the age of 2 months. ${ }^{4,5,9,11}$ In most of these studies, gross motor developpment was persistently delayed up to the age of 2 years. ${ }^{5,9,11}$ At an older age, $39 \%$ and $42 \%$ of 5year-old and school-aged children with CHD, respectively, were shown to experience persistent gross motor deficits. ${ }^{12,13}$ In contrast, some studies revealed an improvement in motor development during infancy. ${ }^{5,9}$ In particular, Mussatto and colleagues reported better motor scores in older CHD children without genetic syndrome, resulting in motor performances similar to those of typically developing infants at the age of 12 months and older. ${ }^{4}$ In their study, $74 \%$ of children received early intervention services from the US regional early intervention programmes or private services, which raised the possibility of a potential benefit of early intervention on motor development.

In an effort to support neurodevelopment in children with CHD and based on the guideline statement of the American Heart Association and the American Academy of Pediatrics, ${ }^{1}$ we established in 2013 an interdisciplinary neurocardiac clinic. We provide early and structured developmental screening including standardized motor assessments at the ages of 4, 12, and 24 months, and older, as well as early individualised intervention sessions to children combined with a psychoeducational support for the parents, tailored to their individual needs.

In a case study, we previously reported significant motor improvement after early intervention in one of our patients with CHD. ${ }^{14}$ However, research is still needed to document the motor development of cohorts of infants enrolled in such structured surveillance programmes and to esti- 
mate the effects of early physical therapy when provided. Therefore, this pilot study describes the developmental trajectory of gross motor skills in infants with CHD aged between 4 and 24 months enrolled in an early and systematic developmental screening programme.

\section{Method}

\subsection{Participants}

All infants with CHD who were referred at birth to the Clinique d'Investigation Neurocardiaque (CINC) of the Sainte-Justine University Hospital Centre, in Montreal, Canada, from March 2013 to June 2016, and who underwent motor evaluations at the ages of 4, 12 and 24 months were considered to be included in this study. Infants were excluded if they: (1) underwent cardiac repair after three months of age as this might have impacted the gross motor functioning at 4 months of age, (2) were diagnosed with a genetic syndrome that affects neurodevelopment, (3) were born preterm (<37 weeks gestational age), or (4) had incomplete assessments or a significant amount of missing data regarding physical therapy. Perinatal, surgical, critical and demographic variables were collected from medical records. Anatomic CHD classification ${ }^{15}$ and surgical risk category ${ }^{16}$ were extracted from the descriptions of cardiac lesions and surgical procedures. This study was conducted with the formal approval of the Research Ethics Board of the CHU Sainte-Justine and all families gave written informed consent for their participation (Ethical review committee: Research Ethics Board of the CHU Sainte-Justine; Approval number: 2015-806, 4024).

\subsection{Gross motor evaluations and intervention}

As part of their developmental follow-up, children's gross motor functioning was assessed using the Alberta Infant Motor Scale (AIMS) ${ }^{17}$ at the age of 4 months \pm 2 weeks, and the gross motor section of the Bayley Scales of Infant and Toddler Development, Third edition (Bayley-III/GM) ${ }^{18}$ at 12 and 24 months \pm 1 month. All motor evaluations were standardized; they were performed at 
4 months by physical therapists (LD and MM), at 12 months by occupational therapists (KG and JP), and at 24 months by a psychologist (MCV).

At 4 months of age, an individualized motor intervention programme was established if needed. Each programme was based on the severity of the impairment as appreciated using the AIMS - a score below the $10^{\text {th }}$ percentile rank being considered as a high-risk score for motor delay - ${ }^{19,20}$ and the physical therapists' observations. If needed, infants received early physical therapy at our neurocardiac clinic before they were referred to their regional paediatric centre at the age of 8 months at the earliest. Physical therapy sessions consisted of strengthening the muscles of the neck, chest and extremities to bring the child to assume and maintain anti-gravity positions such as bringing hands to midline in supine, holding the forearm prop position in prone and maintaining sitting. The sessions also included stimulating transitional movements such as rolling.

Infants of our cohort were divided into three groups according to their motor performance at 4 months and number of physiotherapy sessions received between the ages of 4 and 8 months: the no-intervention group (controls) performed equal or above the $10^{\text {th }}$ percentile rank at the AIMS and received no intervention; the occasional intervention group performed below the $10^{\text {th }}$ percentile rank on AIMS and received one or two therapy sessions; the regular intervention group performed below the $10^{\text {th }}$ percentile rank on AIMS and received a minimum of three sessions. We quantified the number of physical therapy sessions received between 4 and 8 months of age exclusively because of our focus on early motor intervention and because of our inability to document the physiotherapy provided at regional paediatric centres after the age of 8 months.

\subsection{Statistical analyses}

Descriptive statistics were used to characterize the cohort as a whole and to describe each of the groups (no-intervention vs. occasional intervention vs. regular intervention). Frequencies (sample 
size and percentages) were reported for dichotomous and categorical variables. Median and interquartile ranges were reported for continuous variables. We performed a one-way repeatedmeasures analysis of variance (ANOVA) on Bayley-III/GM at 12 and 24 months with the interventional group as an inter-subject factor. Post-hoc tests using pair-wise comparisons were conducted to differentiate gross motor trajectories between the groups. Bonferroni corrections were applied when necessary. Significance level was set at $P<0.05$.

\section{Results and discussion}

\subsection{Sample population}

A total of thirty $(N=30)$ infants were included in this pilot study: six $(n=6)$ infants in the no-intervention group, thirteen $(\mathrm{n}=13)$ in the occasional intervention group, and eleven $(\mathrm{n}=11)$ in the regular intervention group. Perinatal, surgical, critical and demographic characteristics of the cohort are presented in Table 1. The most common heart defects were transposition of the great arteries, coarctation of the aorta, ventricular septal defect, and double outlet right ventricle. The most represented anatomic CHD categories were classes I and II, that is two-ventricle hearts without arch obstruction (66.7\%) and two-ventricle hearts with arch obstruction (20\%). Surgical risk categories 2, 3, and 4 were represented in $10(33.3 \%), 13(43.3)$ and $7(23.3 \%)$ cases, respectively. Median age at cardiac repair was 9.5 days (ICR: 6-26.5). Twenty (66.7\%) infants required both cardiopulmonary bypass $(\mathrm{CPB})$ and cross clamp $(\mathrm{CC})$ during heart surgery, one $(3.3 \%)$ required only $\mathrm{CPB}$, six (20\%) only CC, two (6.7\%) did not require any mechanical support and one $(3.3 \%)$ had missing data. Median paediatric intensive care unit (PICU) and hospital length stays were 5.5 and 19 days (ICR: 4-12.25 and ICR: 10-28). There was no difference in gestational age, birth weight, age at cardiac repair, duration of $\mathrm{CPB}$ or $\mathrm{CC}$ during cardiac surgery, anatomic 
CHD classification, surgical risk category, PICU and hospital length stay between groups. Infants of the no-intervention group had a lower Apgar score at 5 minutes $(P=.009)$.

\subsection{Motor functions and interventions}

Out of 30 infants included in this study, six (20\%) performed above the clinical cut-off at 4 months $(\geq 10$ th percentile rank $)$, and $24(80 \%)$ were at risk of motor delay $\left(<10^{\text {th }}\right.$ percentile rank) and required physical therapy. The proportion of children with gross motor delay at the age of 4 months in our cohort was higher compared to Long and colleagues' study which reported that $36 \%$ of 4-months-olds had delayed gross motor development. ${ }^{11}$ However, our results are overall in agreement with previously reported neurodevelopmental outcomes suggesting that gross motor impairments are common in this population..$^{2,4,5,9}$ These motor impairments may be associated with muscle tone anomalies, core or limb weakness or asymmetries such as a preferential cervical rotation to one side due to plagiocephaly or torticollis. These perturbations likely arise from a complex interplay between prenatal, surgical and environmental risk factors that lead to brain injury and dysfunction. ${ }^{7,21,22}$ In fact, brain injury acquired during the foetal, the postnatal or the perioperative periods, ${ }^{23-27}$ combined with a lack of motor experience, ${ }^{28}$ were shown to contribute to these impairments.

Of the $24(80 \%)$ infants of our cohort who presented gross motor delay and required additional care, $13(54.2 \%)$ received one to two physical therapy sessions between the ages of 4 and 8 months, while $11(45.8 \%)$ received three to six therapies. To the best of our knowledge, no study has quantified early motor interventions in infants with CHD. In older cohorts, the prevalence of children with CHD who used rehabilitation services largely exceeded that of healthy children. ${ }^{29,30}$ Mussatto and colleagues reported that $74 \%$ of 3-year-old infants with CHD received intervention services from US regional early intervention programmes or private therapy, ${ }^{4}$ and $53 \%$ of 5 -year- 
old infants with CHD from the Paris area received at least one rehabilitation service in Calderon and colleagues' study. ${ }^{29}$ Others indicate that $40 \%$ to $95 \%$ of 8 -year-old children with CHD required relevant educational or rehabilitation services, but however they did not received these services. ${ }^{30}$

\subsection{Gross motor trajectory between 12 and 24 months}

The one-way repeated ANOVA revealed a significant increase in Bayley-III/GM scores between the ages of $12(M d n=7, I Q R: 6-9)$ and 24 months $(M d n=9, I Q R: 7-10$; Wilk's Lambda, $\left.F[1,27]=6.233, P=.019, \mathrm{y}_{\mathrm{p}}^{2}=.188\right)$, suggesting an improvement of gross motor skills in our cohort, with scores being comparable to typically developing infants at 24 months of age. A similar gradual increase in motor abilities was previously reported up to 36 months of age in CHD children without genetic syndromes. ${ }^{4,5}$ Interestingly, Mussatto and colleagues observed a gradual improvement in gross motor abilities - which were similar to a normative population at 12 months of age and older - in a cohort of children who received early intervention services in $74 \%$ of cases. ${ }^{4}$ However, they could not determine whether this improvement was the result of a natural evolution or the early intervention following cardiac surgery. Sananes and colleagues did show a gradual improvement in gross motor development from 8 to 24 months of age, but motor skills remained below average. ${ }^{5}$ However, there was no mention of early intervention or of potential access to physical therapy, which may explain the discrepancy with our results.

\subsection{Effect of early individualised motor intervention}

Figure 1 illustrates the trajectories of gross motor development in children with CHD as a function of interventional group between the ages of 4 and 24 months. The ANOVA results revealed a tendency for an effect of group (no-intervention, occasional intervention, and regular intervention) on the Bayley-III/GM scores (Wilk's Lambda, $F[1,27]=3.037, P=.065, \mathrm{y}_{\mathrm{p}}{ }^{2}=.184$ ). Pairwise comparisons showed a marginally significant difference in gross motor functioning between the 
no-intervention and regular intervention groups $(P=.051)$ at the age of 12 months. At this age, only one $(17 \%)$ infant of the no-intervention group scored below normative values compared to six $(55 \%)$ infants of the regular intervention group. This discrepancy reflects the initial gross motor impairment of infants in the regular intervention group as they were still receiving motor stimulation in regional paediatric centres after 8 months of age.

We found a significant increase in Bayley-III/GM scores between $12(M d n=6, I Q R: 2-7)$ and $24(M d n=9, I Q R:$ 6-10) months in the regular intervention group $(P=0.001)$, but no significant change for the no-intervention and occasional intervention groups $(P=0.753$, and $P=$ 0.096). Therefore, infants with motor delay at 4 months who received regular interventions showed improved gross motor scores from 12 to 24 months of age, with their performances being no different than the no-intervention group at 24 months $(P=1.00)$. Although at a trend level, these results suggest a benefit of early physical therapy on the developmental trajectory of gross motor skills in CHD children requiring early corrective surgery and at risk of developmental motor delay.

Very few studies have investigated the effect of interventions to improve neurodevelopmental outcome in children with $\mathrm{CHD}^{31-33}$ and none of these studies addressed gross motor development. Nevertheless, literature on children at risk of impairment, such as preterm or lowbirth-weight infants, brings growing evidence of the positive influence of early intervention on neurodevelopmental outcome. ${ }^{34-36}$ A recent review and meta-analysis on preterm infants highlights the benefits of systematic interventions specifically targeting motor development, such as home or clinic-based motor exercises, and early physical therapy, compared to generic interventions. ${ }^{35}$ Furthermore, interventions received before 12 months of age showed benefits up to the age of 24 months in preterm infants. ${ }^{37}$ The programmes reviewed were offered to all infants regardless of their initial motor abilities; our patient-centred approach differs in that it provides 
psychoeducational support to parents and additional physical intervention sessions only as needed. Interventions that were shown to be the most effective at improving preterm infants' motor skills included supervising the child in practicing movements in supine, prone and side-lying positions, as well as facilitating hands to midline, which are taught in our clinic. ${ }^{35}$ Finally, a recent study of our interdisciplinary team revealed that the ability to maintain the prone prop position in 4-months old infants with CHD resulted in an earlier onset of walking. ${ }^{28}$ Altogether, these findings bring additional support to the idea that early motor experience may influence later motor development in high-risk populations and represents a modifiable factor that should be considered by health care professionals to optimize neurodevelopmental outcome.

\subsection{Limitations}

In an effort to reliably estimate the effects of early motor intervention, we attempted to control potentially cofounding factors and minimize the missing data using conservative inclusion/ exclusion criteria. For instance, we excluded infants who underwent cardiac repair after three months of age or had incomplete assessments. As a result, this study relied on a small sample size, which affects our statistical power and limits the generalizability of our results. These criteria may also have resulted in an over-representation of the most severe cases that may have been more likely to come back for developmental screening. Finally, this study focused on interventions provided to infants with CHD aged between 4 and 8 months of age at our clinic but could not estimate services provided later at regional paediatric centres.

In summary, this pilot study described the trajectory of gross motor development in infants with CHD included in an early and systematic developmental screening programme. We showed an improvement in gross motor development from 4 to 24 months of age in our patient group. Regular physiotherapy sessions seemed to help to improve gross motor skills in infants at risk of 
motor delay. Although at a trend level, these results may suggest a beneficial effect of participating in such an early and structured programme. They also highlighted the importance of performing the early motor skills' surveillance in infants with CHD who underwent cardiac repair in order to identify early those with a delay in development to provide individualized interventions. Large clinical trials are needed to confirm findings and further quantify the benefits of early intervention.

\section{Acknowledgments}

The authors warmly thank all the participating families, as well as the staff of the Clinique d'Investigation Neurocardiaque (CINC) of the CHU Sainte-Justine, Montreal, QC, Canada.

\section{Funding sources}

This work was supported by the Heart and Stroke Foundation of Canada under grant number G16-00012606, the Fonds de la Recherche du Québec - Santé (FRQS) under grant number 28811, and the Canadian Institutes of Health Research (CIHR) under grant number NI203422 held by AG.

\section{Declaration of interest}

The authors declared no interests which may be perceived as posing a conflict or bias. 


\section{References}

1. Marino BS, Lipkin PH, Newburger JW, Peacock G, Gerdes M, Gaynor JW, Mussatto KA, Uzark K, Goldberg CS, Johnson WH, Li J, Smith SE, Bellinger DC, Mahle WT. Neurodevelopmental Outcomes in Children With Congenital Heart Disease : Evaluation and Management: A Scientific Statement From the American Heart Association. Circulation. 2012;126:1143-1172.

2. Limperopoulos C, Majnemer A, Shevell MI, Rohlicek C, Rosenblatt B, Tchervenkov C, Darwish HZ. Predictors of developmental disabilities after open heart surgery in young children with congenital heart defects. The Journal of Pediatrics. 2002;141:51-58.

3. Hallioglu O, Gurer G, Bozlu G, Karpuz D, Makharoblidze K, Okuyaz C. Evaluation of Neurodevelopment Using BayleyIII in Children with Cyanotic or Hemodynamically Impaired Congenital Heart Disease. Congenital Heart Disease. 2015;10(6):537-541.

4. Mussatto KA, Hoffmann RG, Hoffman GM, Tweddell JS, Bear L, Cao Y, Brosig C. Risk and Prevalence of Developmental Delay in Young Children With Congenital Heart Disease. Pediatrics. 2014;133(3):1-8.

5. Sananes R, Manlhiot C, Kelly E, Hornberger LK, Williams WG, Macgregor D, Buncic R, Mccrindle BW. Neurodevelopmental Outcomes After Open Heart Operations Before 3 Months of Age. Annals of Thoracic Surgery. 2012;93(5):1577-1583.

6. Long SH, Galea MP, Eldridge BJ, Harris SR. Performance of 2-year-old children after early surgery for congenital heart disease on the Bayley Scales of Infant and Toddler Development, Third Edition. Early Human Development. 2012;88:603-607.

7. Majnemer A, Limperopoulos C, Shevell MI, Rohlicek C, Rosenblatt B. A New Look at Outcomes of Infants With Congenital Heart Disease. Pediatric Neurology. 2009;40(3):197-204.

8. Bellinger DC, Wypij D, Kuban KC, Rappaport LA, Hickey PR, Wernovsky G, Jonas RA, Newburger JW. Developmental and neurologic status of children at 4 years of age after heart surgery with hypothermic circulatory arrest or low-flow cardiopulmonary bypass. The New England journal of medicine. 1999;332(9):549-555.

9. Cheatham SL, Carey H, Chisolm JL, Heathcock JC. Early Results of Neurodevelopment Following Hybrid Stage I for Hypoplastic Left Heart Syndrome. Pediatric Cardiology. 2015;36:685-691.

10. Nattel SN, Adrianzen L, Kessler EC, Andelfinger G, Dehaes M, Côté-Corriveau G, Trelles MP. Congenital Heart Disease and Neurodevelopment: Clinical Manifestations, Genetics, Mechanisms, and Implications. Canadian Journal of Cardiology. 2017;33:1543-1555.

11. Long SH, Harris SR, Eldridge BJ, Galea MP. Gross motor development is delayed following early cardiac surgery Cardiology in the Young. 2012;22:574-82.

12. Majnemer A, Limperopoulos C, Shevell M, Rosenblatt B, Rohlicek C, Tchervenkov C. Long-term neuromotor outcome at school entry of infants with congenital heart defects requiring open-heart surgery. The Journal of Pediatrics. 2006;148(1):72-77.

13. Holm I, Fredriksen PM, Fosdahl MA, Olstad M. Impaired Motor Competence in School-aged Children With Complex Congenital Heart Disease. Archives of Pediatrics and Adolescent Medicine. 2007;161(10):8-10.

14. Gallagher A, Dagenais L, Doussau A, Décarie J-C, Materassi M, Gagnon K, Prud'homme J, Vobecky S, Poirier N, Carmant L. Significant motor improvement in an infant with congenital heart disease and a rolandic stroke: The impact of early intervention. Developmental Neurorehabilitation. 2017;20(3):165-168.

15. Clancy RR, Mcgaurn SA, Wernovsky G, Spray TL, Norwood WI, Jacobs ML, Murphy JD, Gaynor JW, Goin JE. Preoperative Risk-of-Death Prediction Model in Heart Surgery with Deep Hypothermic Circulatory Arrest in the Neonate. Journal of Thoracic and Cardiovascular Surgery. 2000;119(2):347-357.

16. Jenkins KJ, Gauvreau K. Center-specific differences in mortality: Preliminary analyses using the Risk Adjustment in Congenital Heart Surgery (RACHS-1) method. Journal of Thoracic and Cardiovascular Surgery. 2002;124(1):97-104.

17. Piper MC, Darrah J. Motor Assessment of the Developing Infant. Philadelphia, PA: Saunders; 1994.

18. Bayley N. Bayley Scales of Infant and Toddler Development (3rd edition). San Antonio, TX: Pearson; 2006.

19. Darrah J, Piper M, Watt MJ. Assessment of gross motor skills of at-risk infants: predictive validity of the Alberta Infant Motor Scale. Developmental Medicine and Child Neurology. 1998;40:485-491.

20. Albuquerque PL, Farias Guerra MQ, Carvalho Lima M, Eickmann SH. Concurrent validity of the Alberta Infant Motor Scale to detect delayed gross motor development in preterm infants : A comparative study with the Bayley Concurrent validity of the Alberta Infant Motor Scale to detect delayed gross motor development in pre. Developmental Neurorehabilitation. 2018;21(6):1-7.

21. Birca A, Vakorin VA, Porayette P, Madathil S, Chau V, Seed M, Doesburg SM, Blaser S, Nita DA, Sharma R, Duerden EG, Hickey EJ, Miller SP, Hahn CD. Interplay of brain structure and function in neonatal congenital heart disease. Annals of Clinical and Translational Neurology. 2016;3(9):708-722.

22. Long SH, Physio B, Eldridge BJ, Physio B, Galea MP, Harris SR. Risk Factors for Gross Motor Dysfunction in Infants With Congenital Heart Disease. Infants \& Young Children. 2011;24(3):246-258.

23. Brossard-Racine M, Du Plessis AJ, Vezina G, Robertson R, Bulas D, Evangelou IE, Donofrio M, Freeman D, Limperopoulos C. Prevalence and spectrum of in utero structural brain abnormalities in fetuses with complex congenital 
heart disease. American Journal of Neuroradiology. 2014;35(8):1593-1599.

24. Khalil A, Suff N, Thilaganathan B, Hurrell A, Cooper D, Carvalho JS. Brain abnormalities and neurodevelopmental delay in congenital heart disease: Systematic review and meta-analysis. Ultrasound in Obstetrics and Gynecology. 2014;43:1424.

25. Barrett CS, Bratton SL, Salvin JW, Laussen PC, Rycus PT, Thiagarajan, Ravi R. MBBS M. Neurological injury after extracorporeal membrane oxygenation use to aid pediatric cardiopulmonary resuscitation. Pediatric Critical Care Medicine. 2009;10(4):445-451.

26. Boucek K, Smith C, Olsen M, Argueta-Morales R, DeCampli WM. Risk Factors for Early Neurological Injury in Infants Requiring Deep Hypothermic Circulatory Arrest and/or Selective Cerebral Perfusion. Circulation. 2016;128.

27. Polito A, Barrett CS, Rycus PT, Favia I, Cogo PE, Thiagarajan RR. Neurologic Injury in Neonates with Congenital Heart Disease During Extracorporeal Membrane Oxygenation. ASAIO Journal. 2015;61:43-48.

28. Dagenais L, Materassi M, Desnous B, Vinay M-C, Doussau A, Sabeh P, Prud'homme J, Gagnon K, Lenoir M, Charron M-A, Nuyt AM, Poirier N, Beaulieu-Genest L, Carmant L, Birca A. Superior Performance in Prone in Infants With Congenital Heart Disease Predicts an Earlier Onset of Walking. Journal of Child Neurology. 2018:1-7.

29. Calderon J, Bonnet D, Pinabiaux C, Jambaqué I, Angeard N. Use of early remedial services in children with transposition of the great arteries. The Journal of Pediatrics. 2013;163:1105-1110.

30. Majnemer A, Mazer B, Lecker E, Carter AL, Limperopoulos C, Shevell M, Rohlicek C, Rosenblatt B, Tchervenkov C. Patterns of use of educational and rehabilitation services at school age for children with congenitally malformed hearts. Cardiology in the Young. 2008;18:288-296.

31. Fredriksen PM, Kahrs N, Blaasvaer S, Sigurdsen E, Gundersen O, Roeksund O, Norgaand G, Vik JT, Soerbye O, Ingjer F, Taulow E. Effect of physical training in children and adolescents with congenital heart disease. Cardiology in the Young. 2000;10:107-114.

32. McCusker CG, Doherty NN, Molloy B, Rooney N, Mulholland C, Sands A, Craig B, Stewart M, Casey F. A controlled trial of early interventions to promote maternal adjustment and development in infants born with severe congenital heart disease. Child: care, health and development. 2009;36(1):110-117.

33. McCusker CG, Doherty NN, Molloy B, Rooney N, Mulholland C, Sands A, Craig B, Stewart M, Casey F. A Randomized Controlled Trial of Interventions to Promote Adjustment in Children With Congenital Heart Disease Entering School and Their Families. Journal of Pediatric Psychology. 2012;37(10):1089-1103.

34. Orton J, Spittle A, Doyle L, Anderson P, Boyd R. Do early intervention programmes improve cognitive and motor outcomes for preterm infants after discharge? A systematic review. Developmental Medicine and Child Neurology. 2009;51:851-859.

35. Hughes AJ, Redsell SA, Glazebrook C. Motor Development Interventions for Preterm Infants : A Systematic Review and Meta-analysis. Pediatrics. 2016;138:1-13.

36. Spittle A, Treyvaud K. The role of early developmental intervention to influence neurobehavioral outcomes of children born preterm. Seminars in Perinatology. 2016;40:542-548.

37. Wu Y, Leng C, Hsieh W, Hsu C, Chen WJ, Gau SS, Chiu N, Yang M, Fang L, Hsu H, Yu Y, Wu Y, Chen L-C, Jeng S-F. A randomized controlled trial of clinic-based and home-based interventions in comparison with usual care for preterm infants: Effects and mediators. Research in Developmental Disabilities. 2014;35:2384-2393. 
a)

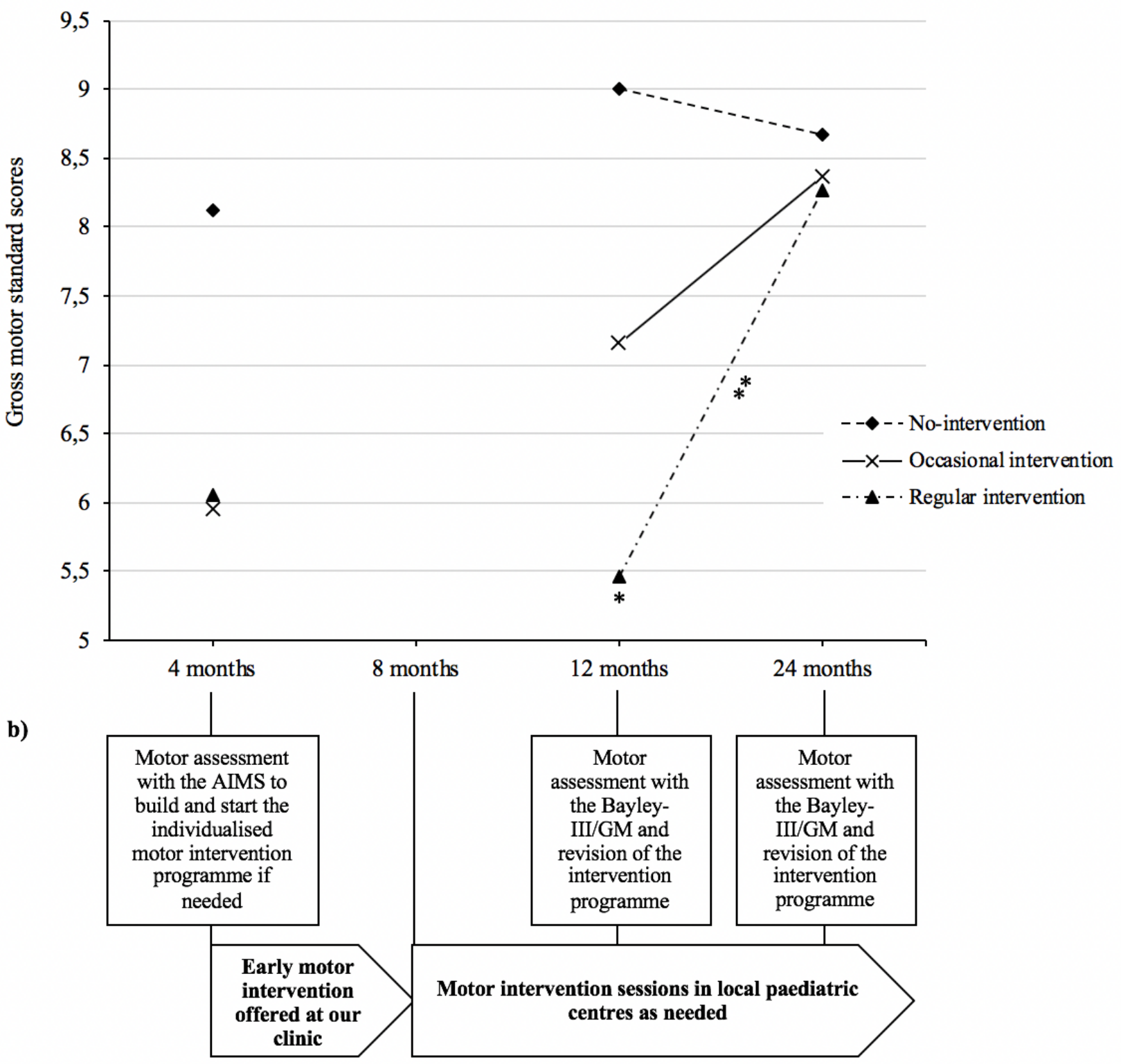

Figure 1. a) Developmental gross motor trajectory from 4 to 24 months of age in groups of infants with CHD: nointervention group (control; dotted line), occasional intervention group (plain line), and regular intervention group (dashed line). Gross motor functions were evaluated with the AIMS at 4 months (percentile ranks converted into standard scores), and with Bayley-III/GM at 12 and 24 months.

b) Timeline of standardised motor assessments and individualised intervention at the CHU Sainte-Justine Clinique d'Investigation Neurocardiaque (CINC).

* indicates statistical trend to significant differences between no-intervention and regular intervention groups $(P$ $=.051)$

** indicates significant increase in gross motor performances for the regular intervention group $(P=.001)$ 
Table 1. Clinical and demographical characteristics of infants with congenital heart disease (CHD). Frequencies (sample size and percentages) were reported for dichotomous and categorical variables. Median and interquartile ranges were reported for continuous variables.

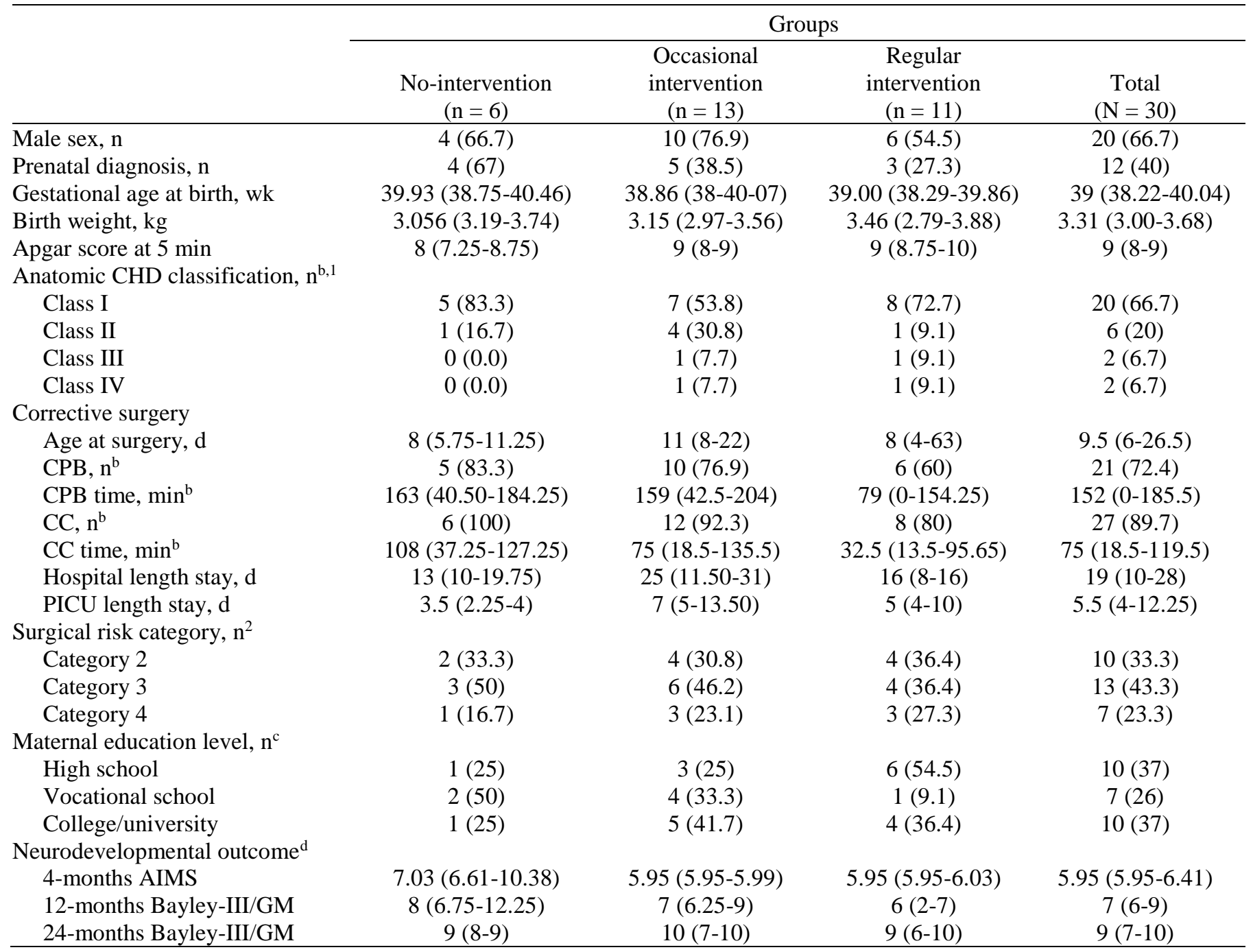

Abbreviations: CHD, congenital heart disease; CPB, cardiopulmonary bypass; CC, cross clamp; PICU, paediatric intensive care unit; AIMS, Alberta Infants Motor Scales; Bayley-III/GM, gross motor scale of the Bayley Scales of Infant and Toddler Development, Third edition.

${ }^{\mathrm{b}}$ One missing data in the intervention group.

${ }^{c}$ Two missing data in the no-intervention group, one missing data in the occasional intervention group.

$\mathrm{d}$ Neurodevelopmental outcome is expressed as standard scores.

1. Clancy RR, Mcgaurn SA, Wernovsky G, Spray TL, Norwood WI, Jacobs ML, Murphy JD, Gaynor JW, Goin JE. Preoperative Risk-of-Death Prediction Model in Heart Surgery with Deep Hypothermic Circulatory Arrest in the Neonate. Journal of Thoracic and Cardiovascular Surgery. 2000;119(2):347-357.

2. Jenkins KJ, Gauvreau K. Center-specific differences in mortality: Preliminary analyses using the Risk Adjustment in Congenital Heart Surgery (RACHS-1) method. Journal of Thoracic and Cardiovascular Surgery. 2002;124(1):97-104. 